\title{
Chronic administration of myristic acid improves hyperglycaemia in the Nagoya-Shibata-Yasuda mouse model of congenital type 2 diabetes
}

\author{
Tamae Takato $^{1} \cdot$ Kai Iwata $^{1} \cdot$ Chiaki Murakami $^{1} \cdot$ Yuko Wada $^{1} \cdot$ Fumio Sakane $^{1}$
}

Received: 7 March 2017 / Accepted: 6 June 2017 /Published online: 13 July 2017

(C) Springer-Verlag GmbH Germany 2017

\begin{abstract}
Aims/hypothesis Previously, we demonstrated that myristic acid (14:0) increases levels of diacylglycerol kinase (DGK) $\delta$, a key enzyme involved in type 2 diabetes exacerbation, and enhances glucose uptake in $\mathrm{C} 2 \mathrm{C} 12$ myotube cells. Moreover, results from a population-based cohort study suggest that consumption of high-fat dairy products, which contain high amounts of myristic acid, is associated with a lower risk of developing type 2 diabetes. Taken together, we hypothesised that intake of myristic acid reduces type 2 diabetes risk in vivo. The aim of this study was to examine the glucoselowering effect of myristic acid in Nagoya-Shibata-Yasuda (NSY) mice, a spontaneous model for studying obesityrelated type 2 diabetes.

Methods Male NSY mice were orally administered vehicle $(n=9), 300 \mathrm{mg} / \mathrm{kg}$ of myristic acid $(n=14)$ or $300 \mathrm{mg} / \mathrm{kg}$ of palmitic acid (16:0) $(n=9)$ every other day from 4 weeks of age. Glucose and insulin tolerance tests were performed at weeks 18, 24 and 30, and weeks 20 and 26, respectively. DGK $\delta$ levels were measured in skeletal muscle from 32-36-week-old NSY mice via western blot.

Results Chronic oral administration of myristic acid ameliorated glucose tolerance (24-28\% decrease in blood glucose levels during glucose tolerance tests) and reduced insulin-
\end{abstract}

Tamae Takato and Kai Iwata contributed equally to this work.

Electronic supplementary material The online version of this article (doi:10.1007/s00125-017-4366-4) contains peer-reviewed but unedited supplementary material, which is available to authorised users.

Fumio Sakane

sakane@ faculty.chiba-u.jp

1 Department of Chemistry, Graduate School of Science, Chiba University, 1-33 Yayoi-cho, Inage-ku, Chiba 263-8522, Japan responsive blood glucose levels ( $\sim 20 \%$ decrease) in male NSY mice compared with vehicle and palmitic acid groups at 24-30 weeks of age (the age at which the severity of type 2 diabetes is exacerbated in NSY mice). Myristic acid also attenuated the increase in body weight seen in NSY mice. Furthermore, the fatty acid increased DGK $\delta$ levels ( 1.6-fold) in skeletal muscle of NSY mice.

Conclusions/interpretation These results suggest that the chronic oral administration of myristic acid improves hyperglycaemia by decreasing insulin-responsive glucose levels and reducing body weight, and that the fatty acid accounts for the diabetes protective properties of high-fat dairy products. Myristic acid is a potential candidate for the prevention and treatment of type 2 diabetes mellitus and its related diseases.

Keywords Dairy fat · Diabetes · Diacylglycerol kinase · Hyperglycaemia $\cdot$ Myristic acid
Abbreviations
DGK Diacylglycerol kinase
NSY Nagoya-Shibata-Yasuda

\section{Introduction}

Type 2 diabetes mellitus is a serious worldwide health issue $[1,2]$. The characteristic features of type 2 diabetes include insulin resistance, glucose intolerance, hyperglycaemia and hyperinsulinaemia [3]. It is known that the glucose-induced insulin resistance is associated with a long-term increase in the intracellular diacylglycerol mass [4].

Diacylglycerol is converted to phosphatidic acid by diacylglycerol kinase (DGK) [5-9]. Ten mammalian DGK isozymes 
are divided into five groups based on their primary structures. The type II DGK subfamily consists of $\delta[10,11], \eta[12,13]$ and $k$ [14] isoforms. In addition, alternative splice variants of DGK $\delta(\delta 1$ and $\delta 2)[11]$ and $\eta(\eta 1$ and $\eta 2)$ [13] have been identified.

DGK $\delta$ is abundantly expressed in the skeletal muscle [10], which is a major target organ for insulin for glucose intake [15]. Recently, Chibalin et al revealed that decreased levels of DGK $\delta$ are closely linked to high glucose-induced insulin resistance, which increases the severity of type 2 diabetes [16]. Moreover, Miele et al demonstrated that acute exposure to high levels of glucose activated DGK $\delta$ in L6 skeletal muscle cells followed by attenuation of protein kinase $\mathrm{C} \alpha$ activity and transactivation of the insulin receptor signal [17]. Furthermore, we recently revealed that DGK $\delta$ preferentially phosphorylates palmitic acid (16:0)-containing diacylglycerol species such as 30:0-, 30:1-, 32:0-, 32:1-, 34:0- and 34:1-diacylglycerol (X:Y, where $\mathrm{X}$ is the total number of carbon atoms and $\mathrm{Y}$ is the total number of double bonds in both acyl chains of the lipids) derived from the phosphatidylcholinespecific phospholipase C pathway in high glucosestimulated C2C12 myoblasts [18].

Because DGK $\delta$ is a key enzyme in the suppression of the pathogenesis of type 2 diabetes as described above, upregulation of DGK $\delta$ is important for prevention and treatment of the disease. Low-intensity endurance exercise increases the expression of DGK $\delta$ proteins in the skeletal muscles of individuals with type 2 diabetes [19, 20]. Interestingly, we recently demonstrated that myristic acid (14:0) substantially enhanced DGK $\delta$ content in $\mathrm{C} 2 \mathrm{C} 12$ myotubes, whereas other fatty acids examined, such as lauric (12:0), palmitic (16:0) and stearic (18:0) acids, did not [21]. Moreover, myristic acid significantly increased glucose uptake in $\mathrm{C} 2 \mathrm{C} 12$ myotubes in a DGK $\delta$-dependent manner [22].

Intriguingly, Ericson et al recently found that the consumption of high-fat dairy products was associated with a lower risk of developing diabetes in a population-based cohort study [23]. It is noteworthy that dairy fat exceptionally contains a lot of myristic acid (approximately $12 \%$ of total fatty acids). Whereas, fat from the other sources, such as meat and plants, is generally less than $1 \%$ myristic acid. Indeed, a significant association between high intake of myristic acid and decreased type 2 diabetes risk was observed [23].

Therefore, we hypothesised that intake of myristic acid reduces type 2 diabetes risk in vivo. However, very little data concerning the effects of myristic acid on animal models of diabetes are available. In the present study, we examined the effect of myristic acid on diabetic risk factors, such as hyperglycaemia and insulin resistance, and DGK $\delta$ expression in Nagoya-Shibata-Yasuda (NSY) mice, which are widely used as a model for type 2 diabetes [24].

\section{Methods}

Materials Myristic acid (14:0) and palmitic acid (16:0) in the form of NEFAs (more than $99 \%$ purity) were purchased from Wako Pure Chemical Industries (Osaka, Japan). BSA (fatty acid free) was obtained from Sigma-Aldrich (St Louis, MO, USA). The fatty acids were dispersed in $10 \%$ (wt/vol.) of BSA solution (vehicle) by sonication to a final concentration of $30 \mathrm{mg} / \mathrm{ml}$.

Animals Male NSY mice [24] (RBRC02946, RIKEN BioResource Center, Tokyo, Japan) were housed individually in stainless wire mesh cages in a room with a $12 \mathrm{~h} \mathrm{light/dark}$ cycle and a constant temperature of $24 \pm 1^{\circ} \mathrm{C}$. Four-week-old male NSY mice were randomly divided into three groups using simple randomisation and orally administered either $10 \%$ (wt $/ \mathrm{vol}$.) of BSA (vehicle, $n=9$ ), $300 \mathrm{mg} / \mathrm{kg}$ of palmitic acid $(n=9)$ or $300 \mathrm{mg} / \mathrm{kg}$ of myristic acid $(n=14)$. These dietary supplements were provided through a stomach tube each morning between 09:00 and 12:00 hours every other day for 28 weeks. All mice were allowed free access to water and chow (Oriental Yeast, Tokyo, Japan) during the experimental period. Calculated values of energy intakes from chow, BSA and fatty acid (myristic or palmitic acid) were: chow, $\sim 63 \mathrm{~kJ} /$ day; BSA, $\sim 0.33 \mathrm{~kJ} /$ day (approximately $0.5 \%$ of chow); fatty acid (myristic or palmitic acid), $\sim 0.21 \mathrm{~kJ} /$ day (approximately $0.3 \%$ of chow). Therefore, the values from chow and BSA with and without fatty acid (myristic or palmitic acid) are $\sim 63.09 \mathrm{~kJ}$ and $\sim 63.30 \mathrm{~kJ}$ (difference: approximately $0.3 \%$ ), respectively. Body weight was measured throughout the experiment (once every 4 weeks). Food intake (for 6 days) was measured at about 28 weeks of age. Group assignment and outcome assessment were blind to the investigator. This study was conducted in compliance with the National Institutes of Health: Guide for the Care and Use of Laboratory Animals, and the Institutional Animal Care and Use of the University of Chiba approved the protocol.

Glucose tolerance test IPGTTs were performed at weeks 18, 24 and 30 . Glucose ( $2 \mathrm{~g} / \mathrm{kg}$ body weight, Wako Pure Chemical Industries) was administered to overnight (16 h)-fasted mice by i.p. injection. Blood samples were collected from the tail vein immediately preceding the glucose injection $(0 \mathrm{~min})$ and 30, 60, 90 and 120 min thereafter. Samples were then centrifuged at $1200 \mathrm{~g}$ for $25 \mathrm{~min}$. Plasma glucose concentrations were measured using a glucose test kit (LabAssay Glucose, Wako Pure Chemical Industries). The AUC of the glucose $(\mathrm{mmol} / \mathrm{l} \times \mathrm{min})$ was also calculated from the glucose measurements at baseline ( $0 \mathrm{~min}), 30,60,90$ and $120 \mathrm{~min}$. Mice were excluded from the datasets if they were non-responsive: if they had a blood glucose levels $<13.3 \mathrm{mmol} / \mathrm{l} 30 \mathrm{~min}$ after glucose injection. 
Insulin tolerance test Insulin tolerance tests were performed at weeks 20 and 26. Following $16 \mathrm{~h}$ of food deprivation, an insulin solution $(0.5 \mathrm{U} / \mathrm{kg}$ body weight, Novolin, Novo Nordisk, Tokyo, Japan) was administered i.p. Blood samples were obtained from the tail vein immediately prior to the insulin administration ( $0 \mathrm{~min}$ ), and 15, 30, 45 and $60 \mathrm{~min}$ later. Samples were then centrifuged at $1200 \mathrm{~g}$ for $25 \mathrm{~min}$. Plasma glucose concentrations were measured using a glucose test kit (LabAssay Glucose, Wako Pure Chemical Industries). The AUC of glucose $(\mathrm{mmol} / 1 \times \mathrm{min})$ was also calculated from the glucose measurements at baseline ( $0 \mathrm{~min}), 15,30,45$ and $60 \mathrm{~min}$. Mice were excluded from the datasets if they were non-responsive: if blood glucose concentrations were not decreased $15 \mathrm{~min}$ after insulin injection.

Blood insulin concentration Blood samples were obtained via the tail vein and then centrifuged at $1200 \mathrm{~g}$ for $25 \mathrm{~min}$. Plasma insulin concentrations were measured by an insulin ELISA kit (Mouse Insulin ELISA kit, Morinaga Institute of Biological Science, Tokyo, Japan).

Western blot analysis Skeletal muscle from 32-36-week-old male NSY mice was homogenised in lysis buffer $(50 \mathrm{mmol} / \mathrm{l}$ HEPES, pH 7.2, 150 mmol/1 NaCl, 5 mmol/1 $\mathrm{MgCl}_{2}$ ) containing $1 \mathrm{mmol} / \mathrm{l}$ phenylmethylsulfonyl fluoride, $20 \mu \mathrm{g} / \mathrm{ml}$ each of leupeptin, pepstatin, aprotinin and soybean trypsin inhibitor, and Complete EDTA-free Protease Inhibitor Cocktail (Roche Applied Science, Penzberg, Germany), and centrifuged at $3000 \mathrm{~g}$ for $15 \mathrm{~min}$. The protein concentration in the supernatant fractions was determined using a bicinchoninic acid protein assay kit (Thermo Scientific, Hudson, NH, USA). The tissue lysates $(50 \mu \mathrm{g}$ of protein) were separated on SDSPAGE, and the separated proteins were transferred to a polyvinylidene difluoride membrane (Pall Life Sciences, Port Washington, NY, USA). The membrane was blocked with 5\% skimmed milk (wt/vol.) and incubated with a rabbit anti-DGK $\delta$ polyclonal antibody $(1: 1000)$ (which was previously validated $[10,11])$, overnight at $4^{\circ} \mathrm{C}$. The immunoreactive bands were visualised using a peroxidase-conjugated goat anti-rabbit IgG antibody (1:20,000; Jackson ImmunoResearch Laboratories, West Grove, PA, USA) and the ECL Western Blotting Detection System (GE Healthcare Bio-Sciences, Piscataway, NJ, USA) according to the manufacturers' instructions. To control for background staining, the western blotting procedure was carried out on all membranes with the omission of the anti-DGK $\delta$ antibody (data not shown).

Statistical analysis Data were analysed using PRISM version 5 (GraphPad Software, La Jolla, CA, USA) and expressed as mean \pm SEM. Comparisons of two groups were performed using an unpaired Student's $t$ test. Comparisons of more than two groups were performed by one-way ANOVA followed by the Tukey's post hoc test. The results were considered to be significant if the value of $p$ was $<0.05$.

\section{Results}

Effect of myristic acid on plasma glucose levels in NSY mice High levels of fasting plasma glucose were observed in the male NSY mice after 24 weeks of age [25]. We first determined longitudinal changes in fasting plasma glucose levels in the mice at 4-32 weeks of age. As shown in Fig. 1, the fasting plasma glucose levels of the myristic acid group at 24, 28 and 32 weeks of age were clearly lower than the control group and tended to decrease compared with the palmitic acid group.

Since impairment of glucose tolerance in the mice was also detected after 24 weeks of age [25], an IPGTT was performed in mice at 18, 24 and 30 weeks of age. We confirmed that glucose tolerance was chronologically impaired in the control mice (Fig. 2). Glucose tolerance levels in the myristic acid group were not affected at 18 weeks, compared with the control and palmitic acid groups (Fig. 2a). Intriguingly, we found that glucose tolerance was significantly improved at 24 and 30 weeks of age in the myristic acid group compared with the control and palmitic acid groups (Fig. 2c, e). As shown in Fig. $2 \mathrm{~b}, \mathrm{~d}, \mathrm{f}$, the means of AUC for glucose at 24 and 30 weeks of age, but not at 18 weeks, were also markedly reduced by the chronic administration of myristic acid compared with vehicle and palmitic acid administration.

Of the control group, 86-89\% developed diabetes (i.e. the plasma glucose level was $>11.1 \mathrm{mmol} / \mathrm{l}$ after $120 \mathrm{~min}$ in a

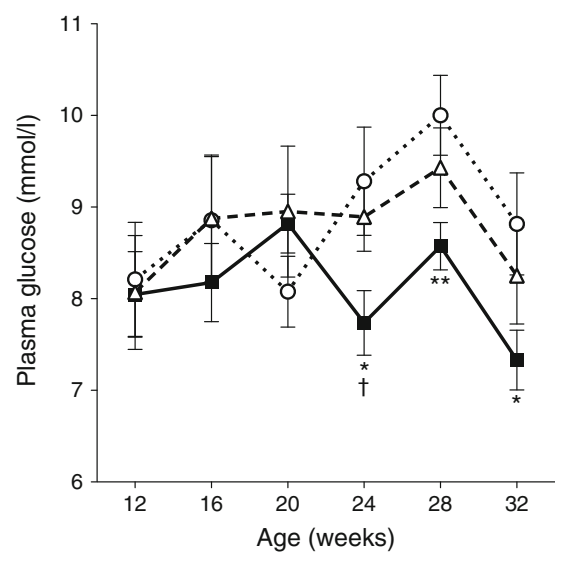

Fig. 1 Effect of myristic acid on fasting blood glucose concentration in male NSY mice. Male NSY mice were orally administered either vehicle (control) (circles/dotted lines), $300 \mathrm{mg} / \mathrm{kg}$ of myristic acid (squares/solid lines) or $300 \mathrm{mg} / \mathrm{kg}$ of palmitic acid (triangles/dashed lines) every other day. Longitudinal analysis of the fasting blood glucose concentration was performed in overnight-fasted NSY mice from 8 to 32 weeks of age. Control, $n=9$; myristic acid, $n=14$; palmitic acid, $n=9$. Data are presented as means \pm SEM. $* p<0.05, * * p<0.01$ vs the control group; ${ }^{\dagger} p<0.05$ vs the palmitic acid group 

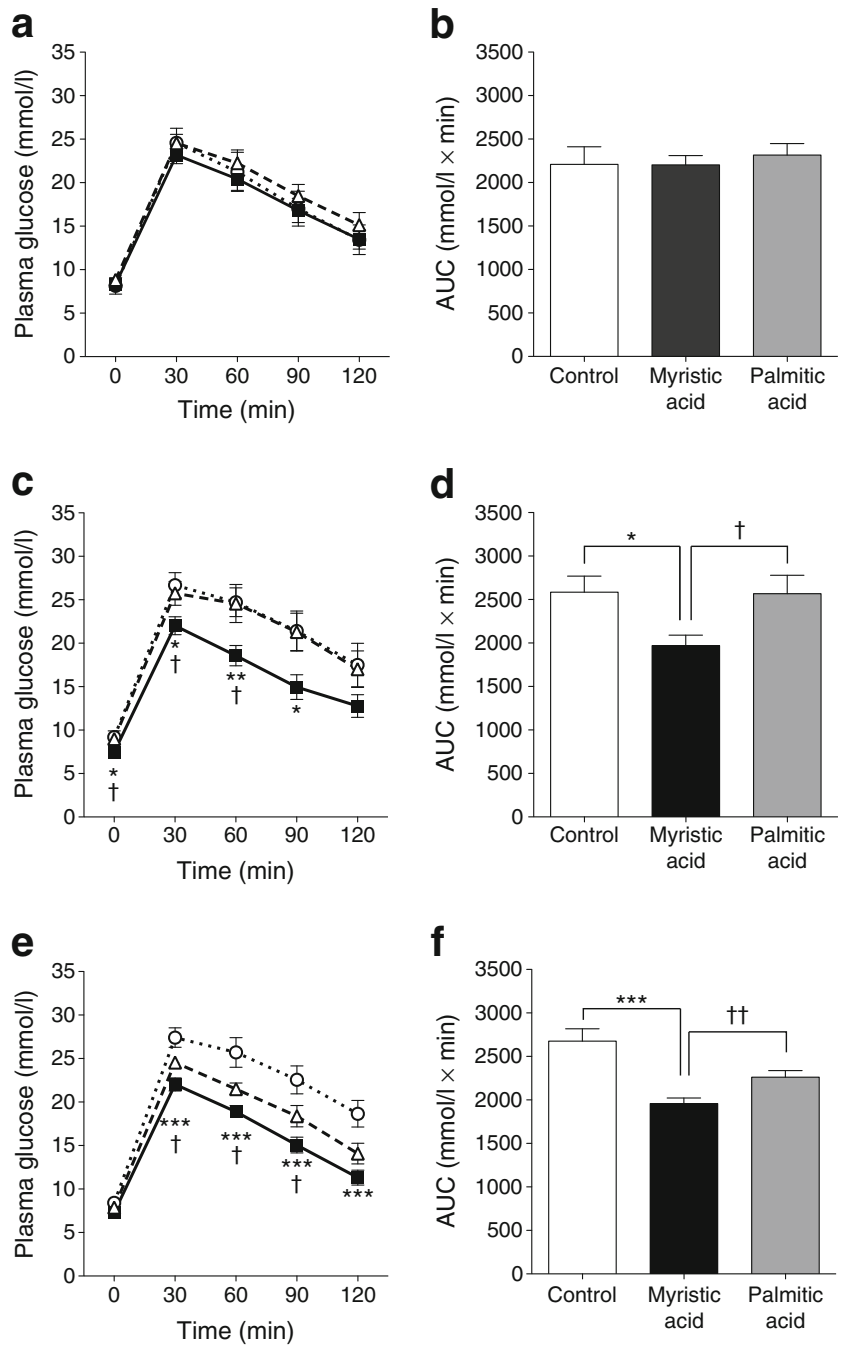

Fig. 2 Effect of myristic acid on glucose tolerance in male NSY mice. Male NSY mice were orally administered either vehicle (control) (circles/ dotted lines), $300 \mathrm{mg} / \mathrm{kg}$ of myristic acid (squares/solid lines) or $300 \mathrm{mg} / \mathrm{kg}$ of palmitic acid (triangles/dashed lines) every other day. Longitudinal analysis of the fasting GTT was performed in overnightfasted male NSY mice at $(\mathbf{a}, \mathbf{b}) 18,(\mathbf{c}, \mathbf{d}) 24$ and $(\mathbf{e}, \mathbf{f}) 30$ weeks of age. (a, c, e) Mean blood glucose and (b, d, f) mean AUC are shown. Control, $n=7-9$; myristic acid, $n=12-14$; palmitic acid, $n=7-9$. Data are presented as means \pm SEM. $* p<0.05, * * p<0.01$, *** $p<0.005$ vs the control group; ${ }^{\dagger} p<0.05,{ }^{\dagger \dagger} p<0.01$ vs the palmitic acid group

GTT) by 24-30 weeks of age (Fig. 3). Of the palmitic acid group, $78-86 \%$ developed diabetes by $24-30$ weeks of age. In contrast, the numbers of mice that developed diabetes at 24 and 30 weeks of age (58\% and $36 \%$ of the total, respectively) were markedly decreased following administration of myristic acid (Fig. 3).

Effect of myristic acid on insulin-responsive plasma glucose levels in NSY mice Insulin resistance was observed in the male NSY mice after 24 weeks of age [25]. Thus, at 20 and 26 weeks the mice were subjected to an insulin tolerance test. We confirmed that insulin resistance was chronologically

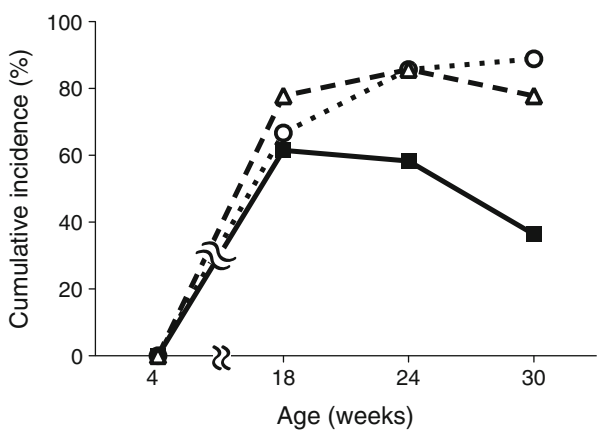

Fig. 3 Effect of myristic acid on cumulative incidence of diabetes. Male NSY mice were orally administered either vehicle (control) (circles/dotted lines), $300 \mathrm{mg} / \mathrm{kg}$ of myristic acid (squares/solid lines) or $300 \mathrm{mg} / \mathrm{kg}$ of palmitic acid (triangles/dashed lines) every other day. Mice were examined at 18, 24 and 30 weeks of age. Control, $n=7-9$; myristic acid, $n=12-14$; palmitic acid, $n=7-9$

worsened (Fig. 4). Plasma glucose levels were not significantly changed in the myristic acid group at 20 weeks of age (Fig. $4 a)$. However, in the myristic acid group at 26 weeks of age, plasma glucose concentrations were significantly lower than in the palmitic acid and control group following a single s.c. administration of insulin (Fig. 4c). However, plasma glucose levels did not differ between the palmitic acid group and the control group. The means of AUC for glucose at 26 weeks of
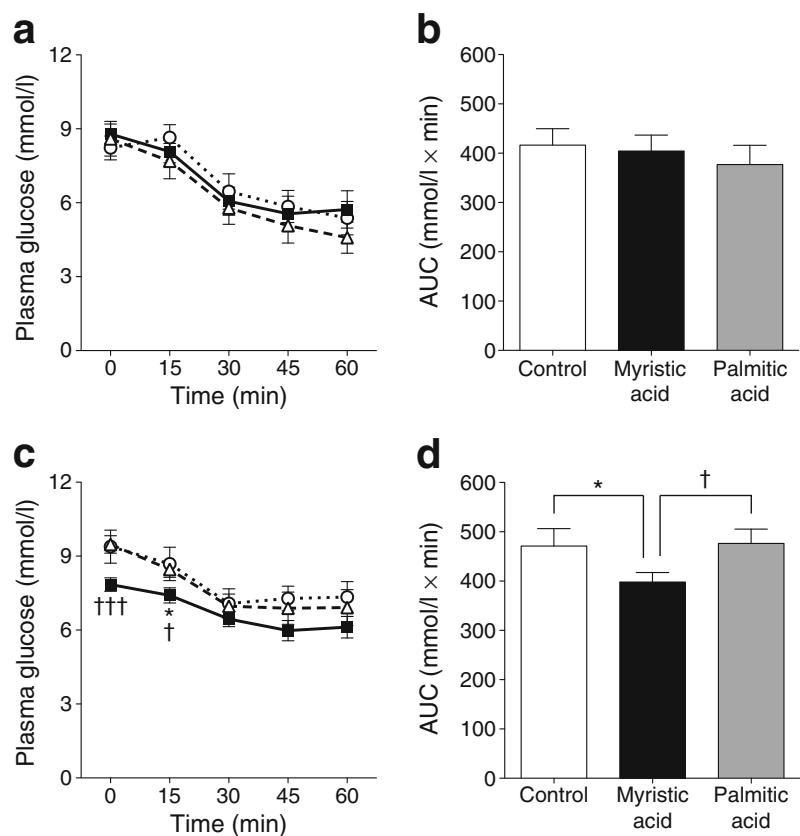

Fig. 4 Effect of myristic acid on insulin tolerance in male NSY mice. Male NSY mice were orally administered either vehicle (control) (circles/ dotted lines), $300 \mathrm{mg} / \mathrm{kg}$ of myristic acid (squares/solid lines) or $300 \mathrm{mg} / \mathrm{kg}$ of palmitic acid (triangles/dashed lines) every other day. Longitudinal analysis of the fasting insulin tolerance test in overnightfasted NSY mice at (a, b) 20 and (c, d) 26 weeks of age was performed. $(\mathbf{a}, \mathbf{c})$ Mean blood glucose and $(\mathbf{b}, \mathbf{d})$ mean AUC are shown. Control, $n=6-7$; myristic acid, $n=11$; palmitic acid, $n=7-8$. Data are means \pm SEM. $* p<0.05$ vs the control group. ${ }^{\dagger} p<0.05,{ }^{\dagger \dagger} p<0.005$ vs the palmitic acid group 
age, but not 20 weeks, were significantly reduced by the chronic administration of myristic acid compared with control and palmitic acid administration (Fig. 4d).

Insulin levels prior to the glucose injection and $30 \mathrm{~min}$ thereafter in the male NSY mice at 24 weeks of age in GTTs (see Fig. 2c) were analysed. As shown in the electronic supplementary material (ESM) Fig. 1, the insulin levels prior to the glucose injection and 30 min thereafter were not significantly changed in the myristic acid group compared with the control and palmitic acid groups. The insulin levels $30 \mathrm{~min}$ after the glucose injection in the palmitic acid group were greater than in the control group.

Effect of myristic acid on body weight As shown in Fig. 5a, the chronic administration of myristic acid to the NSY mice caused a moderate decrease in body weight at 24, 28 and 32 weeks of age compared with the palmitic acid or control groups. In addition, there was a trend towards a reduction in the weight of the epididymal fat pads ( $p=0.141$ vs the control group; $p=0.078$ vs the palmitic acid group) following chronic administration of myristic acid (ESM Fig. 2). However, food intake in the myristic acid group was not significantly
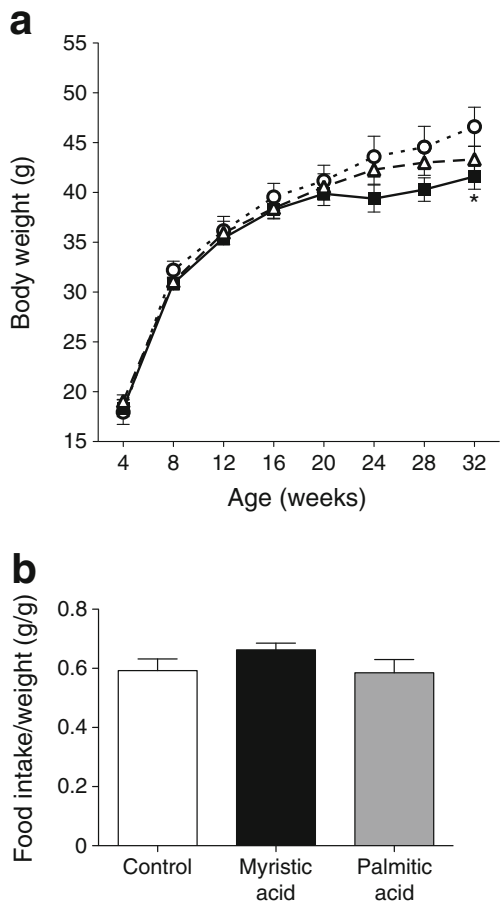

Fig. 5 Effect of myristic acid on the body weight and food intake of male NSY mice. Male NSY mice were orally administered either vehicle (control) (circles/dotted lines), $300 \mathrm{mg} / \mathrm{kg}$ of myristic acid (squares/solid lines) or $300 \mathrm{mg} / \mathrm{kg}$ of palmitic acid (triangles/dashed lines) every other day. (a) Body weight was determined at 4-32 weeks of age. Control, $n=9$; myristic acid, $n=14$; palmitic acid, $n=9$. (b) Food intake (weight of food intake/mouse body weight) was analysed for 6 days at approximately 28 weeks of age. Control, $n=5$; myristic acid, $n=6$; palmitic acid, $n=5$. Data are presented as means \pm SEM. $* p<0.05$ vs the control group different from the control and palmitic acid groups (Fig. 5b), suggesting that the reductions in body and fat pad weight were not caused by lower food intake in the myristic acid group.

We examined whether the organs were affected by the chronic administration of myristic acid. No obvious changes were observed in the dissected organs, including the skeletal muscle, liver, pancreas, stomach and small intestine, from the mice in the myristic acid group (data not shown).

Effect of myristic acid on the expression level of DGK $\delta$ in skeletal muscle Chibalin et al [16] demonstrated that reduction of DGK $\delta$ accelerated the pathogenesis of type 2 diabetes. Moreover, we revealed that myristic acid substantially enhanced the DGK $\delta$ content [21] and significantly increased glucose uptake in a DGK $\delta$-dependent manner [22] in $\mathrm{C} 2 \mathrm{C} 12$ myotubes. Therefore, next we examined whether production of DGK $\delta$ in skeletal muscle was enhanced by chronic administration of myristic acid. Alternative splicing products of DGK $\delta$, DGK $\delta 1(130 \mathrm{kDa})$ and $\delta 2(135 \mathrm{kDa})$, have been identified [11]. We confirmed that DGK $\delta 2(135 \mathrm{kDa})$ is produced in the skeletal muscle in NSY mice (Fig. 6a). We found that the levels of DGK $\delta$ in skeletal muscle were significantly increased (approximately 1.6-fold increase) by chronic administration of myristic acid, but not palmitic acid, compared with the control group (Fig. 6b).
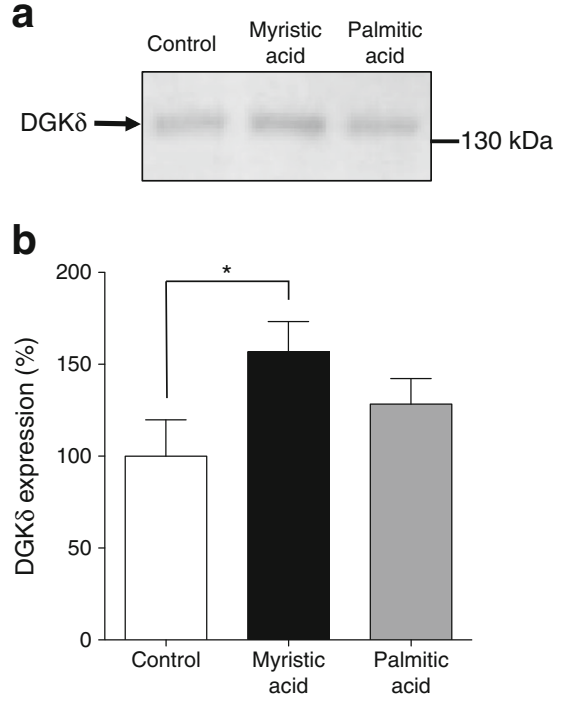

Fig. 6 Effect of myristic acid on DGK $\delta$ levels in skeletal muscle in male NSY mice. Male NSY mice were orally administered either vehicle (control), $300 \mathrm{mg} / \mathrm{kg}$ of myristic acid or $300 \mathrm{mg} / \mathrm{kg}$ of palmitic acid every other day. The level of DGK $\delta$ in skeletal muscle of NSY mice at 32 36 weeks of age was determined by western blotting using an anti-DGK $\delta$ antibody. (a) A representative blot is shown. (b) Amount of DGK $\delta$ protein was quantified by densitometry using Image-J software. $n=6-8$ in each group. Data are presented as means \pm SEM. $* p<0.05$ vs the control group 


\section{Discussion}

Risk factors for type 2 diabetes mellitus include hyperglycaemia and insulin resistance. Our results demonstrate that chronic oral administration of myristic acid improves hyperglycaemia and insulin resistance in spontaneously diabetic male NSY mice.

Ericson et al. recently reported that the consumption of high-fat dairy products was associated with a lower risk of developing diabetes in a population-based cohort study [23]. Dairy fat contains an exceptionally large proportion of myristic acid (approximately 12\% of total fatty acids). Whereas, fat from the other sources, such as meat and plants, is generally less than $1 \%$ myristic acid. Indeed, the authors observed that a high intake of myristic acid (14:0) was significantly associated with a decreased risk of type 2 diabetes, whereas intake of palmitic acid (16:0) was not [23]. The administration of $300 \mathrm{mg} / \mathrm{kg}$ of myristic acid into a mouse every other day is equivalent to an intake of $9 \mathrm{~g}$ of the fatty acid every day for a $60 \mathrm{~kg}$ person. This amount is easily taken by people who willingly eat high-fat dairy products on a daily basis. Therefore, it is possible that myristic acid contributes to the favourable effect of high-fat dairy products on the development of diabetes. Our results support the observations by Ericson et al [23]. However, experimental studies in healthy mice and humans are still missing. This data from mouse models of diabetes should be interpreted carefully.

The administration of myristic acid increased DGK $\delta$ levels (Fig. 6b). DGK $\delta$ has been reported to positively regulate insulin receptor signalling $[16,17]$. Therefore, the favourable effects of myristic acid on hyperglycaemia and insulinresponsive blood glucose levels may be mediated at least in part by upregulation of the DGK $\delta$ in skeletal muscle. However, identifying the detailed mechanisms of the favourable effects in vivo was beyond the scope of this study. Studies to investigate changes in diacylglycerol and phosphatidic acid levels and how they are involved in the activation of conventional/novel and atypical protein kinase $\mathrm{C}$ in skeletal muscle and the liver are warranted. Preliminary results (data not shown) have indicated that diacylglycerol and phosphatidic acid levels are regulated in a complex manner.

Palmitic acid (16:0) slightly reduced blood glucose concentration in a GTT (Fig. 2). However, the effects were weaker and appeared later compared with myristic acid. Palmitoleic acid $(16: 1, n-7)$ has also been reported to be effective on hyperglycaemia in a diabetic mouse model (KK-A $\mathrm{A}^{\mathrm{y}}$ mice) [26]. Neither palmitic nor palmitoleic acid increase DGK $\delta$ expression $[21,22]$. Therefore, these fatty acids probably improve hyperglycaemia in diabetic mice through a different pathway to myristic acid.

Ueda et al reported that the fasting plasma glucose levels of the NSY mice were increased from 24 to 32 weeks of age [24, 25]. We obtained essentially the same result (Fig. 1). In addition, the maximum plasma glucose levels of the NSY mice in a GTT were longitudinally increased from $\sim 22.2$ to $\sim 27.8 \mathrm{mmol} / 1$ from 18 to 30 weeks of age (Fig. 2). Moreover, the number of diabetic NSY mice in which the plasma glucose level was $>11.1 \mathrm{mmol} / \mathrm{l}$ after $120 \mathrm{~min}$ of glucose administration in a GTT was also increased from 18 to 30 weeks of age (Fig. 3). These results suggest that in NSY mice the severity of hyperglycaemia is exacerbated at 24-30 weeks of age. Myristic acid began to show its effects on hyperglycaemia and insulin resistance from 24 weeks of age (Figs 1, 2, 3), suggesting that the fatty acid prevents hyperglycaemia at the exacerbation stage in male NSY mice.

An insulin tolerance test showed that plasma glucose concentrations in the myristic acid group at 26 weeks of age, but not at 20 weeks, were lower than in the palmitic acid and control groups (Fig. 4). The results suggest that myristic acid decreases insulin-responsive blood glucose levels at the point of diabetes exacerbation in NSY mice, as with the effect on hyperglycaemia described above. Myristic acid did not decrease plasma insulin levels in the NSY mice (ESM Fig. 1). Ueda et al reported that plasma insulin levels were not greatly increased in NSY mice even when impaired glucose tolerance was detected [24]. Therefore, it is likely that myristic acid primarily affects insulin receptor signalling rather than insulin secretion in NSY mice. We previously demonstrated that, in addition to insulin-dependent glucose uptake, myristic acid enhanced insulin-independent glucose uptake in $\mathrm{C} 2 \mathrm{C} 12$ mouse myotubes [22]. Therefore, myristic acid might enhance both insulin-dependent and -independent glucose uptake in type 2 diabetic mice.

There is growing evidence that excess body weight is associated with type 2 diabetes $[27,28]$. The administration of myristic acid to NSY mice reduced body weight and fat gain (Fig. 5 and ESM Fig. 2), which may in turn improve lipid and glucose metabolism. Myristic acid treatment did not affect food intake (Fig. 5b), suggesting that certain metabolic changes were induced by myristic acid. However, food intake was only measured for a short period in the present study. Therefore, although it seems unlikely, we cannot exclude the possibility that the differences in body weight are due to unmeasured differences in food intake.

Cumulative evidence supports that fatty acids are involved in type 2 diabetes mellitus. Many reports have shown that fatty acids with different degrees of saturation affect insulin sensitivity and lipid/glucose metabolism differently. Although saturated fatty acids generally cause insulin resistance, unsaturated fatty acids ameliorate the pathology associated with diabetes [29, 30]. NEFAs (especially saturated fatty acids) generally attenuate insulin sensitivity and glucose uptake in skeletal muscle [31, 32]. However, the detailed effects of fatty acid carbon length on hyperglycaemia and insulin resistance have not been thoroughly investigated. In the present study, we have demonstrated that myristic acid (14:0), but not 
palmitic acid (16:0), strongly improves hyperglycaemia and insulin resistance.

In conclusion, the chronic oral administration of myristic acid to NSY mice markedly ameliorated their diabetic condition. Hyperglycaemia, insulin resistance and body weight were all reduced in response to myristic acid. Therefore, myristic acid is a potential candidate for the prevention and treatment of type 2 diabetes mellitus and related diseases. However, the detailed mechanisms of its favourable effects in vivo are still poorly understood. Moreover, it is still not clear what effects myristic acid has in normal mice, such as high-fat-fed wild-type mice. Further studies are needed to clarify these issues.

Acknowledgements We acknowledge D. Takahashi (Graduate School of Science, Chiba University, Chiba, Japan) for a critical review of the manuscript.

Data availability The datasets generated and/or analysed during the current study are available from the corresponding author on reasonable request.

Funding This work was supported in part by grants from MEXT/JSPS KAKENHI grant numbers 17H03650 (Grant-in-Aid for Scientific Research (B)), 22370047 (Grant-in-Aid for Scientific Research (B)), 23116505 (Grant-in-Aid for Scientific Research on Innovative Areas), 25116704 (Grant-in-Aid for Scientific Research on Innovative Areas), 26291017 (Grant-in-Aid for Scientific Research (B)) and 15K14470 (Grant-in-Aid for Challenging Exploratory Research); the Japan Science and Technology Agency (AS221Z00794F, AS231Z00139G, AS251Z01788Q and AS2621643Q); the Naito Foundation; the Hamaguchi Foundation for the Advancement of Biochemistry; the Daiichi-Sankyo Foundation of Life Science; the Terumo Life Science Foundation; the Futaba Electronic Memorial Foundation; the Daiwa Securities Health Foundation; the Ono Medical Research Foundation; the Japan Foundation for Applied Enzymology; the Food Science Institute Foundation; the Skylark Food Science Institute; the Asahi Group Foundation; and the Japan Milk Academic Alliance (FS).

Duality of interest The authors declare that they have no conflicts of interest regarding the content of this article.

Contribution statement TT, KI, CM and YW designed and performed the experiments, and analysed the data. FS conceived and coordinated the study and wrote the paper. All authors drafted the article and revised it critically for important intellectual content and provided final approval of the version to be published. FS is responsible for the integrity of the work as a whole.

\section{References}

1. Guariguata L, Whiting DR, Hambleton I, Beagley J, Linnenkamp U, Shaw JE (2014) Global estimates of diabetes prevalence for 2013 and projections for 2035. Diabetes Res Clin Pract 103:137149

2. Zimmet P, Alberti KG, Shaw J (2001) Global and societal implications of the diabetes epidemic. Nature 414:782-787
3. Biddinger SB, Kahn CR (2006) From mice to men: insights into the insulin resistance syndromes. Annu Rev Physiol 68:123-158

4. Kraegen EW, Saha AK, Preston E et al (2006) Increased malonyl$\mathrm{CoA}$ and diacylglycerol content and reduced AMPK activity accompany insulin resistance induced by glucose infusion in muscle and liver of rats. Am J Phys Endocrinol Metab 290:E471-E479

5. Goto K, Hozumi Y, Kondo H (2006) Diacylglycerol, phosphatidic acid, and the converting enzyme, diacylglycerol kinase, in the nucleus. Biochim Biophys Acta 1761:535-541

6. Merida I, Avila-Flores A, Merino E (2008) Diacylglycerol kinases: at the hub of cell signalling. Biochem J 409:1-18

7. Sakane F, Imai S, Kai M, Yasuda S, Kanoh H (2007) Diacylglycerol kinases: why so many of them? Biochim Biophys Acta 1771:793806

8. Shulga YV, Topham MK, Epand RM (2011) Regulation and functions of diacylglycerol kinases. Chem Rev 111:6186-6208

9. van Blitterswijk WJ, Houssa B (2000) Properties and functions of diacylglycerol kinases. Cell Signal 12:595-605

10. Sakane F, Imai S, Kai M, Wada I, Kanoh H (1996) Molecular cloning of a novel diacylglycerol kinase isozyme with a pleckstrin homology domain and a C-terminal tail similar to those of the EPH family of protein tyrosine kinase. J Biol Chem 271:8394-8401

11. Sakane F, Imai S, Yamada K, Murakami T, Tsushima S, Kanoh H (2002) Alternative splicing of the human diacylglycerol kinase $\delta$ gene generates two isoforms differing in their expression patterns and in regulatory functions. J Biol Chem 277:43519-43526

12. Klauck TM, Xu X, Mousseau B, Jaken S (1996) Cloning and characterization of a glucocorticoid-induced diacylglycerol kinase. J Biol Chem 271:19781-19788

13. Murakami T, Sakane F, Imai S, Houkin K, Kanoh H (2003) Identification and characterization of two splice variants of human diacylglycerol kinase $\eta$. J Biol Chem 278:34364-34372

14. Imai S, Kai M, Yasuda S, Kanoh H, Sakane F (2005) Identification and characterization of a novel human type II diacylglycerol kinase, DGKK. J Biol Chem 280:39870-39881

15. DeFronzo RA, Jacot E, Jequier E, Maeder E, Wahren J, Felber JP (1981) The effect of insulin on the disposal of intravenous glucose. Results from indirect calorimetry and hepatic and femoral venous catheterization. Diabetes 30:1000-1007

16. Chibalin AV, Leng Y, Vieira E et al (2008) Downregulation of diacylglycerol kinase delta contributes to hyperglycemia-induced insulin resistance. Cell 132:375-386

17. Miele C, Paturzo F, Teperino R et al (2007) Glucose regulates diacylglycerol intracellular levels and protein kinase $\mathrm{C}$ activity by modulating diacylglycerol-kinase subcellular localization. J Biol Chem 282:31835-31843

18. Sakai H, Kado S, Taketomi A, Sakane F (2014) Diacylglycerol kinase $\delta$ phosphorylates phosphatidylcholine-specific phospholipase C-dependent, palmitic acid-containing diacylglycerol species in response to high glucose levels. J Biol Chem 289:26607-26617

19. Fritz T, Kramer DK, Karlsson HK et al (2006) Low-intensity exercise increases skeletal muscle protein expression of PPAR $\delta$ and UCP3 in type 2 diabetic patients. Diabetes Metab Res Rev 22: 492-498

20. Teran-Garcia M, Rankinen T, Koza RA, Rao DC, Bouchard C (2005) Endurance training-induced changes in insulin sensitivity and gene expression. Am J Phys Endocrinol Metab 288:E1168E1178

21. Sakiyama S, Usuki T, Sakai H, Sakane F (2014) Regulation of diacylglycerol kinase delta2 expression in $\mathrm{C} 2 \mathrm{C} 12$ skeletal muscle cells by free fatty acids. Lipids 49:633-640

22. Wada Y, Sakiyama S, Sakai H, Sakane F (2016) Myristic acid enhances diacylglycerol kinase delta-dependent glucose uptake in myotubes. Lipids 51:897-903 
23. Ericson U, Hellstrand S, Brunkwall L et al (2015) Food sources of fat may clarify the inconsistent role of dietary fat intake for incidence of type 2 diabetes. Am J Clin Nutr 101:1065-1080

24. Ueda H, Ikegami H, Yamato E et al (1995) The NSY mouse: a new animal model of spontaneous NIDDM with moderate obesity. Diabetologia 38:503-508

25. Ueda H, Ikegami H, Kawaguchi Y et al (2000) Age-dependent changes in phenotypes and candidate gene analysis in a polygenic animal model of type II diabetes mellitus; NSY mouse. Diabetologia 43:932-938

26. Yang ZH, Miyahara H, Hatanaka A (2011) Chronic administration of palmitoleic acid reduces insulin resistance and hepatic lipid accumulation in KK-Ay Mice with genetic type 2 diabetes. Lipids Health Dis 10:120

27. Aucott LS (2008) Influences of weight loss on long-term diabetes outcomes. Proc Nutr Soc 67:54-59
28. Holbrook TL, Barrett-Connor E, Wingard DL (1989) The association of lifetime weight and weight control patterns with diabetes among men and women in an adult community. Int J Obes 13: 723-729

29. Misra A, Singhal N, Khurana L (2010) Obesity, the metabolic syndrome, and type 2 diabetes in developing countries: role of dietary fats and oils. J Am Coll Nutr 29:289S-301S

30. Riserus U, Willett WC, Hu FB (2009) Dietary fats and prevention of type 2 diabetes. Prog Lipid Res 48:44-51

31. Boden G (2003) Effects of free fatty acids (FFA) on glucose metabolism: significance for insulin resistance and type 2 diabetes. Exp Clin Endocrinol Diabetes 111:121-124

32. Roden M, Price TB, Perseghin G et al (1996) Mechanism of free fatty acid-induced insulin resistance in humans. J Clin Invest 97: 2859-2865 